D0I: 10.7213/aurora.27.041.A003 ISSN 0104-4443

Licenciado sob uma Licença Creative Commons

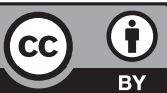

\title{
Hermenéutica de sí y fin de la exomologesis: Michel Foucault y la exclusión de la materialidad de la veridicción en el cristianismo
}

\author{
Hermeneutics of the self and the end of exomologesis: \\ Michel Foucault and the exclusion of the materiality of \\ veridiction in Christianity
}

\begin{abstract}
Agustín Colombo ${ }^{[a, b]^{*}}$
[a] Université Paris 8 Vincennes - Saint-Denis, Saint-Denis, Francia

[b] Universidad de Buenos Aires, Ciudad de Buenos Aires, Argentina

\section{Resumen}

El presente trabajo se focaliza en las investigaciones de Michel Foucault sobre las discontinuidades relativas a la práctica de la confesión (aveu) introducidas en el marco del monacato de los siglos IV y V. En este sentido, se intenta realizar un aporte que tienda a complementar las reflexiones del filósofo francés relacionadas con la conformación de la
\end{abstract}

* AG: doctor, e-mail: agustincolomb082@gmail.com 
"voluntad de saber" en Occidente y en particular en torno al rol central que la confesión (aveu) ocupa en ella. A partir del problema foucaultiano de la "exclusión", abordamos el modo en que la exomologesis - una forma no verbal de decir la verdad del individuo propia del cristianismo de los primeros siglos — deja de ser el medio fundamental de veridicción de sí mismo en el seno de la institución monástica. Así, nuestro análisis nos permitirá abordar aspectos centrales respecto de los cambios introducidos por el monacato en la configuración de las relaciones entre subjetividad y verdad en Occidente.

Palabras Clave: Cristianismo. Confesión. Verdad. Subjetividad.

\section{Abstract}

This article tackles Michel Foucault's researches on the discontinuities that the monasticism of $4^{\text {th }}$ and $5^{\text {th }}$ Centuries introduced in the practice of confession (aveu). Focusing on the central role played by confession, our aim is to complement with a new perspective the French philosopher's inquiries regarding the conformation of the "will to know" in Western society. We start from the Foucaultian problem of exclusion and we use it as a guideline in order to analyze how the exomologesis - a non-verbal way of saying the truth typical of the first centuries of Christianity - loses its fundamental role as a way of self-veridiction in the context of early monasticism. In this way, our work approaches crucial issues about the monasticism-derived changes in the configuration of the relationships between subjectivity and truth in the Western society.

Keywords: Christianity. Confession. Truth. Subjectivity.

\section{Introducción}

La morfología de la voluntad de saber en Occidente ha sido una línea de reflexión constante en las investigaciones de Michel Foucault. Se podría decir, sin dudas, que aunque este término sea más frecuente en los trabajos de los años 1970, podemos observar la presencia de este campo de problematización desde los primeros escritos del filósofo hasta sus últimos trabajos. 
En cuanto respecta a sus trabajos sobre el Cristianismo, en los años 1970 Foucault asigna a un conjunto de técnicas de la "pastoral cristiana" un lugar absolutamente capital respecto a la configuración de ciertos ámbitos vinculados a las ciencias humanas, como por ejemplo la psiquiatría o el psicoanálisis. En efecto, entre estas técnicas podemos ubicar la confesión (aveu) que en el primer volumen de la Historia de la sexualidad es concebida ni más ni menos que cómo la "matriz general que rige la producción del discurso verdadero sobre el sexo" (FOUCAULT, 2012 [1976], p. 84)․․ De esta manera, la confesión funciona como un engranaje insustituible en lo que Foucault denomina los dispositivos de "alianza" y de "sexualidad", dos conceptos clave que permitirán al filósofo explicar la producción de la sexualidad en Occidente al mismo tiempo que desarticular lo que él mismo denomina "hipótesis represiva" como explicación de nuestra sexualidad.

Ahora bien, tanto en La voluntad de saber cómo en el curso en el Collège de France del año 1974-1975, Los Anormales, el horizonte histórico de la investigación foucaultiana sobre el vínculo entre las técnicas de la pastoral cristiana y los campos modernos del conocimiento se limita al análisis de una serie de manuales de confesores que vieron la luz con posterioridad a la Contrarreforma católica puesta en marcha por el Concilio de Trento.

En este artículo nos concentraremos en un momento absolutamente fundamental en la historia de la confesión (aveu) pero cambiando el horizonte de análisis de las reflexiones de Foucault sobre el tema. En este sentido, abordaremos los trabajos que el filósofo ha dedicado a los primeros siglos del cristianismo a los fines de detenernos en ciertos elementos indispensables que nos permitan comprender la preeminencia de la confesión (aveu) como forma de decir la verdad en Occidente. Nos detendremos entonces en el análisis de dos prácticas centrales, a saber, la exomologesis y la exagoreusis.

1 Para el presente trabajo han sido utilizados sólo los textos en lengua francesa, por este motivo las traducciones al español han sido realizadas por el autor. Sin embargo, en la bibliografía, a continuación de las versiones francesas de las fuentes consultadas, se citan las traducciones españolas actualmente existentes. 
Así, la exploración de estas dos maneras de decir la verdad que Foucault aborda en el marco de sus trabajos de los años 1980 será guiada por las siguientes preguntas: ¿Cuáles son las principales cuestiones que ponen en juego la desaparición de la exomologesis como forma preeminente de decir la verdad de sí mismo?¿Qué desplazamientos vinculados a los objetos de análisis de Foucault cabe señalar a fin de precisar lo que se encuentra en juego en la ruptura introducida por el monacato respecto al rol central de una manifestación de sí no verbal?

Enunciando estas preguntas, el objeto de este trabajo intenta emplazarse bajo la línea inaugural de la actividad de investigación de Foucault en el Collège de France. En este sentido, trataremos de ubicarnos en lo que en 1970 era anunciado como un "teatro muy provisorio" (FOUCAULT, 2012 [1971], p. 10) y de interrogarnos en qué medida la desaparición de la exomologesis como forma privilegiada de la veridicción de sí mismo puede ser leída como una exclusión que determina los modos de relacionarnos con la verdad.

Para ello, el trabajo será dividido en tres momentos. En primer lugar, nos detendremos en el análisis de las principales características de la exomologesis, para ser más preciso, trataremos de abordar su aspecto "material" como forma de decir la verdad. En segunda instancia y a fin de considerar la exclusión de la exomologesis, será necesario estudiar ciertos aspectos de la exagoreusis y de la práctica de la confesión (aveu) en el marco del universo monástico de los siglos IV y V. Finalmente, a modo de conclusión, consideraremos algunas de las principales problemáticas que suscita el abordaje de la exomologesis en términos de "exclusión".

\section{La exomologesis y la manifestación material de la verdad del penitente}

La primera mención en las investigaciones actualmente publicadas de Foucault relativa a una manifestación no verbal de la verdad en el conjunto de las técnicas cristianas aparece hacia mediados de los años 1970. Durante la lección del 19 de febrero de 1975, en el marco de un análisis sobre la penitencia en el cristianismo primitivo, Foucault 
evoca un ritual de la penitencia caracterizado por el aspecto público de los actos. Según explica el filósofo, en ese entonces, el ritual de la penitencia estaba definido por la manifestación de la voluntad del pecador de adquirir el status de penitente, status que era otorgado por el obispo en medio de una ceremonia pública. Una vez celebrada esta ceremonia, el penitente entraba en el orden de la penitencia, lo que implicaba la realización de una serie de actos como "portar el cilicio, hábitos especiales, la interdicción de los cuidados de limpieza [...] la no participación de los sacramentos, en todo caso, de la comunión, la imposición de ayunos rigurosos, la interrupción de todo tipo de relación sexual" (FOUCAULT, 1999, p. 159).

No obstante la constatación de esta breve mención de la práctica penitencial en Los Anormales, podemos decir sin dudas, que el estudio más detallado de una manifestación de la verdad de sí no asimilable a la confesión (aveu) en el cristianismo fue emprendido por Foucault a partir de los años 1980, justamente en ocasión del análisis de la penitencia canónica.

En la lección del 5 de marzo de 1980 del curso Del gobierno de los vivos (Du gouvernement des vivants), Foucault delimita en el interior de la penitencia canónica, tres actos de manifestación de la verdad, o mejor dicho, dos procedimientos de exteriorización de la verdad propios de la penitencia y un "acto de verdad" que funciona como una especie de umbral, dado que constituye el momento absolutamente precedente de la entrada al status penitencial. Esta manifestación de la verdad que Foucault individualiza particularmente a partir de sus lecturas de San Cipriano es denominada expositio casus. Se trata de una instancia de veridicción liminar en la que el pecador declara verbalmente al obispo y de manera privada los pecados que ha cometido con el objeto de obtener un cambio de estado, a saber, el estado de penitente.

En cambio, en el interior mismo de la penitencia, el filósofo francés circunscribe una forma de exteriorización no verbal de la verdad que denomina exomologesis a fines de marcar su especificidad y de mantener la diferencia con el vocablo latín confessio. Así, debemos entender el término exomologesis como "la manifestación de su acuerdo, el reconocimiento, el hecho de que uno admite algo, a saber, su pecado y 
que se es pecador. Es eso, en resumen, la exomologesis que se le requiere al penitente" (FOUCAULT, 2012a, p. 197).

Ahora bien, la exomologesis o exomologèse está constituida por una ambivalencia puesto que ella implica a la vez un acto preciso y una instancia más global relativa al status de penitente. En cuanto acto concreto, la exomologesis consiste en una manifestación espectacular de sí que tenía lugar durante el tiempo penitencial, en el umbral del templo y justo antes de la reconciliación del pecador. En este sentido, el ejemplo tal vez más paradigmático y detallado de este ritual Foucault lo encuentra en las Cartas de San Jerónimo. Nos referimos al caso de Fabiola, quién bajo los ojos de la ciudad de Roma, durante los días que precedían la Pascua, lloraba "con los cabellos dispersos, el rostro pálido, las manos desarregladas, la cabeza manchada de cenizas y humildemente inclinada" mientras que se lastimaba su pecho y su rostro y mostraba a todos "sus heridas y su cuerpo empalidecido" (FOUCAULT, 2012b, p. 106).

Este ritual tenía por objeto manifestar el hecho de que el penitente reconocía que era un pecador, es decir, que no era cuestión aquí de exteriorizar el pecado en sí mismo. La acción reprobada no era objeto del procedimiento penitencial.

Pero en tanto instancia global, la exomologesis implicaba en cambio la penitencia completa. Este alcance de la palabra es individualizado en particular por Foucault en la lectura que realiza del capítulo 9 de De paenitentia de Tertuliano. Aquí, la interpretación del vocablo latín habitus parece jugar un rol absolutamente relevante en el análisis foucaultiano. En efecto, en lugar de limitar el campo semántico al sentido de hábito en cuanto vestimenta, conforme la traducción propuesta por Pierre de Labriolle - que según Philippe Chevallier sería el traductor de la edición de De paenitentia consultada por Foucault (CHEVALLIER, 2011, p. 197) - el filósofo entiende que habitus "es mucho más verosímilmente el modo de vida en general". De este modo, la exomologesis comprendería "una manera de vivir y no solamente una manera de vestirse" (FOUCAULT, 2012a, p. 204).

En este sentido, Foucault señala que según Tertuliano, este modo de vida implica entre otras cosas "que uno se acueste bajo el saco y la 
ceniza, que se envuelva el cuerpo de oscuros harapos" que se practique "un beber y un comer simples", que el penitente alimente sus plegarias "con sus ayunos", que él gima, llore y muja, día y noche frente a Dios, su Señor (FOUCAULT, 2012a, p. 204-205).

De esta manera, entre la exomologesis como acto concreto y específico que se desarrolla antes del momento de la reconciliación y la exomologesis - modo de vida que abarca el status global del penitente habría una relación de intensificación: el ritual concreto no sería otra cosa que la intensificación, la dramatización en un momento específico de lo que era el drama permanente de toda la penitencia.

A diferencia de la expositio casus, es decir, la exteriorización juridico-verbal y concreta al obispo de las faltas cometidas, los procedimientos penintenciales propiamente dichos en el marco de la penitencia canónica, se caracterizaban por su aspecto público, por el hecho de que ponían en juego una serie de elementos expresivos no verbales que en tanto tales no tenían ninguna función analítica o descriptiva y que manifestaban la verdad del pecador a partir de los ritos y de su vida misma, sin necesidad de hacer funcionar una instancia en la que tuvieran que ser manifestadas las faltas cometidas.

Puesto que ella ponía en juego la verdad del pecador en tanto tal, es decir, en tanto que individuo que efectivamente había pecado, la exomologesis constituye entonces para Foucault una "tentación ontológica del cristianismo" (FOUCAULT, 2013, p. 89) que se desarrolla enteramente en el elemento de una publicación no verbal de sí mismo o de una publicatio sui en términos de Tertuliano. Es esta capacidad de manifestar la verdad del penitente a partir de la vida misma que él lleva, que nos permite remarcar el aspecto "material" de esta forma de decir la verdad propia de la penitencia canónica. Son los gestos, la vida misma - y no la oralidad - las instancias que ponen de manifiesto la verdad del individuo que los practica.

Ahora bien, tal como lo señala Foucault, la historia de la penitencia será la historia de la manera en la que la instancia jurídico verbal y exterior a la penitencia - la expositio casus - será introducida al interior de la penitencia y se sustituirá completamente a la publicación de sí (FOUCAULT, 2012a, p. 206). Este momento constituye 
para el diagnóstico foucaultiano un episodio absolutamente central para la historia de las relaciones entre subjetividad y verdad en Occidente, no solamente porque la verdad del pecador podrá ser problematizada de ahora en adelante a partir de un modelo jurídico, sino también porque para la subjetividad occidental este momento implica una relación con el discurso y con la confesión (aveu) muy propia de nuestra civilización.

En las siguientes líneas de este análisis nos concentraremos entonces en un momento bien específico de esta historia relativa a la "victoria de la confesión (aveu)" sobre otros procedimientos penitenciales. Se trata de un momento en el que Foucault constata la emergencia de la necesidad de la palabra en el seno de las técnicas de la vida monástica. De este modo, abordaremos los análisis foucaultianos realizados en torno al nacimiento de una hermenéutica de sí durante los siglos IV y V, centrados particularmente en la lectura de los textos de Casiano. Sin embargo, tal como hemos aclarado al inicio de este trabajo, se tratará de considerar este acontecimiento relativo al origen de la hermenéutica de sí en función de la discontinuidad que él implica en relación a los procedimientos penitenciales propios de la penitencia canónica. Esto nos permitirá observar de una mejor manera los motivos por los que esta necesidad hermenéutica ocupa un lugar determinante en la historia de la verbalización de los procedimientos penitenciales. Dicho de otro modo, en el próximo apartado intentaremos examinar por qué y de qué manera se puede considerar que las técnicas monásticas contribuyen a la exclusión de una forma de decir la verdad de sí mismo que opera a partir de los actos y de la forma de vida que el cristiano lleva.

\section{La exagoreusis y el nacimiento de la hermenéutica de sí}

¿De qué manera será entonces "desarticulada" la exomologesis? La respuesta a esta pregunta debemos sin dudas buscarla no ya en la penitencia, sino en el marco de la dirección espiritual y en particular en 
la instauración de las reglas de obediencia del mundo monástico de la Antigüedad Tardía².

Según Foucault, el núcleo de la dirección monástica está constituido por el acoplamiento o la articulación entre dos reglas: la verbalización exhaustiva y la obediencia completa. En este sentido, para el filósofo estas reglas nos permiten no sólo abordar las características principales de la dirección espiritual monástica sino que también permiten introducirnos en la importancia que reviste el monacato en tanto momento genético fundamental de la subjetividad:

Decir todo de sí mismo, no esconder nada, no querer nada para sí mismo, obedecer en todo; la conjunción entre estos dos principios está, creo, en el corazón mismo, no solamente de la institución monástica cristiana, sino de toda una serie de prácticas, de dispositivos que van a conformar lo que constituye la subjetividad cristiana y, por lo tanto, la subjetividad occidental (FOUCAULT, 2012a, p. 260).

Ahora bien, para obedecer completamente, hay que aprender a desarrollar la capacidad de mirarse a sí mismo, de hacer de sí mismo un objeto de análisis. En otros términos, es necesario aprender a examinarse a sí mismo. Esta relación entre el examen de sí mismo y la obediencia está destinada en Casiano a conjurar dos peligros susceptibles de afectar la vida del monje: de un lado el peligro de relajamiento que podría llevarlo a "las más grandes debilidades"; y del otro lado, el peligro de exceso de ascesis en el que podrían confundirse "la demasiada confianza en sí mismo, el orgullo, la vanidad o el deseo de deslumbrar al otro" (FOUCAULT, 2012a, p. 284-285). Al principio que apunta a garantizar el equilibrio que evite el exceso, Casiano le da el nombre de discretio.

Como explica Foucault, la palabra discretio deriva del término

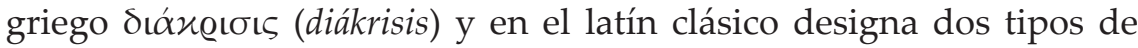
actividad. En primer lugar, la facultad de distinguir, la capacidad de

2 Si bien la cuestión de la obediencia reviste una importancia central en la instauración de la confesión ( $a v e u$ ), ella implica a su vez un campo de exploración (en especial el del gobierno de los otros y el de los efectos de subjetivación) que excede claramente las líneas de este trabajo. En este sentido, circunscribimos nuestro análisis a las modificaciones que introduce el monacato respecto de las relaciones entre el sujeto y su propia verdad. 
separar lo que se encuentra en exceso y de encontrar la justa división que permitirá trazar una "línea recta entre los dos peligros". Pero en segundo lugar, el término evoca una actividad del orden del juicio. A partir de ella, uno puede colocarse en el medio y "ver lo que está de más o lo que no es suficiente" (FOUCAULT, 2012a, p. 285). En este sentido, la discretio no comporta ninguna novedad respecto de la Antigüedad, puesto que ella ya era un tema de la sabiduría antigua. La diferencia que introduce Casiano consiste en que, según el monje, el hombre no puede servirse de la discretio, puesto que ella no funcionará como garantía de la mesura del exceso.

A diferencia del sabio antiguo, quien apoyado sobre su logos podía practicar "una división entre lo que era demasiado y lo que era demasiado poco" (FOUCAULT, 2012a, p. 289), el cristiano en el marco del monacato es aquel a quién le falta la capacidad de poner en práctica por sus propios medios una acción que le permita medir aquello que es o excesivo o insuficiente. Este acontecimiento absolutamente fundamental respecto de las relaciones consigo mismo en la historia de Occidente es explicado por Foucault a partir de dos argumentos, uno de orden coyuntural y otro de orden teológico.

En tanto cuestión coyuntural, el problema de la ausencia de discretio en el hombre debe ser comprendido teniendo en cuenta la necesidad de poner un límite al exceso de ascetismo. En los ejemplos dados por Casiano, Foucault subraya la presencia permanente de esta cuestión, lo que le permite concluir que la organización monástica tiene una "punta anti-ascética" (FOUCAULT, 2012a, p. 287). En efecto, a fines de comprender el surgimiento del monacato, Foucault entiende que es necesario tener presente que la institución monástica ha guardado siempre una relación de ambigüedad respecto del ascetismo. En este sentido, si bien en el corazón de las prácticas monásticas encontramos elementos ascéticos, se trata siempre de un ascetismo institucionalizado que pone en juego "estructuras de poder muy fuertes" (FOUCAULT, 2012b, p. 124).

Respecto de las razones de orden teológico, el elemento que explica esta relación replegada del individuo consigo mismo y sobre sí mismo, caracterizada por la imposibilidad de la auto-indexación de la 
mesura, Foucault lo encuentra en una teología de la presencia del diablo en el hombre característica de Casiano y por otro lado típica de los autores de la época a la que el monje pertenecía.

Así, a partir del análisis de los textos de Casiano, Foucault distingue tres elementos centrales de esta presencia satánica en el hombre. En primer lugar, ella es perpetua, esto quiere decir que ella no será jamás conjurada. En segundo lugar, la presencia del diablo tiene lugar en el interior del sujeto mismo, no en su alma, sino en el interior de su "subjetividad" (FOUCAULT, 2012a, p. 290). De este modo y puesto que el alma del hombre es de la misma naturaleza que el espíritu del mal, el cuerpo del individuo constituye una especie de sede compartida donde coexisten su alma y el demonio. Se trata así de una "co-posesión" (FOUCAULT, 2012a, p. 291). Finalmente, el tercer elemento - que Foucault no circunscribe en cuanto tal, pero cuya individualización se hace posible en función de su importancia - , el cuerpo es el ámbito en el que el espíritu del mal actuará sobre el alma del individuo. Instalado en el cuerpo, el diablo desplegará su accionar a través del engaño y del error. Esta presencia del diablo en el hombre marca los términos de una relación consigo mismo caracterizada por una opacidad que el cristiano no podrá jamás disipar por sus propios medios.

Ahora bien, justamente esta falta de discretio será colmada por la función de la confesión exhaustiva (aveu) y su lugar capital en el marco del examen de sí monástico. A partir de la verbalización, será posible practicar una separación que permitirá establecer el origen de los pensamientos. La confesión (aveu) será entonces "un operador de discriminación" (FOUCAULT, 2012a, p. 299) ? $^{3}$ qe pone en funcionamiento una serie de mecanismos por el simple hecho de hablar. En primer lugar, a partir del "criterio de la vergüenza" (FOUCAULT, 2012a, p. 299) porque si los pensamientos son de mala ley tenderán a esconderse, se resistirán a ser exteriorizados y de este modo quién practica la confesión sentirá el rechazo de hablar. En segundo lugar, por razones de orden "cosmo-teológico" (FOUCAULT, 2012a, p. 299) los pensamientos de

3 Foucault insistirá en esta capacidad de discriminación de la confesión también en Mal faire, dire vrai (FOUCAULT, 2012b, p. 149). 
origen satánico pueden vivir sólo en la oscuridad de las tinieblas y no podrán jamás soportar la luz que implica su manifestación por medio de la palabra. Finalmente, el simple hecho de hablar pone en práctica una "expulsión material" (FOUCAULT, 2012a, p. 299). Las capacidades de la palabra son en este sentido asimiladas a un acto de exorcismo que tiene el objeto de hacer salir los pensamientos pecaminosos que se esconden en el individuo.

Esta necesidad de una confesión incesante, de decir todo desde el fondo de los pensamientos al momento mismo de la gestación de las ideas, es denominada por Foucault "exagoreusis", palabra que para el filósofo designa "la perpetua puesta en discurso de sí mismo" (FOUCAULT, 2012a, p. 301, 2012b, p. 161, 2013, p. 87).

De este modo, el monacato marca una ruptura fundamental en relación a una forma de manifestar la verdad de sí mismo como la exomologesis en donde la verbalización - tal como lo hemos visto precedentemente - no cumplía ninguna función relativa a la transmisión de la verdad del individuo. En el monacato, la palabra será el garante del conocimiento de la verdad de los arcana del monje por medio de la interpretación de los pensamientos que la confesión misma pone en marcha. En el marco de una línea de análisis en términos de verdicción y subjetividad, se pasa entonces de una exteriorización indudable y manifiesta de la verdad de sí a través de los gestos y de la vida misma, al nacimiento de un nuevo problema en el cual el pronunciamiento mismo de la palabra ocupa un lugar central gracias a su función hermenéutica.

Dada la importancia de este acontecimiento en el marco general de una historia de las relaciones entre subjetividad y verdad en Occidente, es de capital importancia señalar aquí una fundamental diferencia entre la función de la confesión (aveu) en el marco del monacato y la manera de operar de este modo de veridicción en el campo de las Ciencias Humanas.

En este sentido - y aunque Foucault sea lo suficientemente claro es necesario insistir sobre la tarea específica de separación y de discriminación de la verdad de sí que cumple la confesión (aveu) en el monacato. En el marco de la institución monacal, y como se desprende de 
la lectura de Casiano que realiza Foucault, la confesión misma opera el diagnóstico relativo al origen de los pensamientos. Como hemos visto, el sólo hecho de hablar permite establecer la proveniencia de los pensamientos sobre la base de una serie de criterios. No habría entonces una actividad complementaria de interpretación por parte del monjeguía en la relación de dirección espiritual a partir de la cual se pueda establecer el lugar de gestación de los logismoi.

Esta autosuficiencia hermenéutica de la confesión constituye una diferencia fundamental a los fines de comprender las discontinuidades entre la técnica de confesión cristiana tal como la concibe el monacato de los siglos IV y V, y el funcionamiento de esta "matriz general" de producción de la verdad de la sexualidad en Occidente en el campo de las Ciencias Humanas modernas.

En efecto, entre uno de los mecanismos que es posible individualizar a los fines de comprender de qué manera la confesión se inserta y cumple una serie de roles determinantes en ciertos campos científicos en el Occidente moderno, debemos considerar el método de la interpretación. En este sentido, la conexión entre el procedimiento de la confesión (aveu) de origen cristiano y la discursividad científica es posible gracias a la combinación entre la verbalización de sí mismo y la validación científica que realiza quién recoge e interpreta la confesión (aveu). De este modo, la verdad de sí para ser constituida en tanto verdad científica debe ser necesariamente doblada:

[La verdad] no reside en el sujeto que, confesándose la llevaría a la luz, ya completamente realizada. Ella se constituye por partida doble: presente, pero incompleta, ciega a ella misma en la persona misma que habla, ella no puede realizarse sino en quién la recibe. Es este último quién dirá la verdad de esta verdad oscura. Hay que doblar la revelación de la confesión a través del desciframiento de lo que ella dice. Quien escucha no será simplemente el dueño (maître) del perdón, el juez que condena o absuelve; él será el dueño (maître) de la verdad. Su función es hermenéutica (FOUCAULT, 2012 [1976], p. 89).

Evidentemente, de Casiano a Heinrich Kaan o a Freud habrá pasado mucho tiempo y se habrán producido otros acontecimientos 
que darán forma a nuestra voluntad de saber, específicamente sobre el sexo. A modo de simple mención, nos permitimos evocar aquí el lugar central que ocuparán para Foucault los manuales de los confesores en la época de la Contrarreforma. En ese período se constituye todo un foco de interrogación y de análisis relativo al cuerpo a partir del cual se abordarán los deseos, los pensamientos, las sensaciones y los placeres. El cuerpo surgirá así como un objeto de examen privilegiado y esta manifestación somática de los placeres producirá un juego hermenéutico en torno a un "código carnal" (FOUCAULT, 1999 p. 173).

Pero volviendo al objeto de este trabajo, podemos afirmar que la función hermenéutica de la confesión (aveu) de origen monástico es un advenimiento que marcará la desaparición de la exomologesis como forma de veridicción central en la que se manifiesta la verdad del individuo de una manera no verbal y de un modo incontestable. En este sentido, si este acontecimiento marca un momento fundamental en la historia de la práctica de la confesión (aveu) y constituye a su vez una suerte de a priori capital relativo a la constitución de las Ciencias Humanas, a partir del análisis que venimos desarrollando podemos observar de qué manera la emergencia de la hermenéutica como función de la confesión (aveu) implica la exclusión de una forma de veridicción de sí mismo que pone en juego la materialidad de los gestos y de la vida misma que con anterioridad al monacato ocupaba un lugar central entre las prácticas penitenciales.

Ahora bien, llegados a este punto de nuestro análisis, resulta conveniente hacernos una pregunta: ¿Cuáles son las principales cuestiones que están en juego en la exclusión de esta forma no verbal de decir la verdad sobre sí mismo en el marco de un diagnóstico que atañe la conformación de nuestra voluntad de saber?

\section{Conclusión: Algunas cuestiones en torno a la exclusión de la exomologesis}

En su curso en el Collège de France de 1970-1971, Lecciones sobre la voluntad de saber, Foucault tendrá la posibilidad de precisar una de las líneas de trabajo que había anunciado algunos meses antes, en el 
marco de su lección inaugural en esa misma institución. Se trata de una línea de exploración vinculada con las determinaciones de la producción discursiva. Más concretamente, según Foucault, la producción discursiva en nuestras sociedades estaría marcada por una serie de sistemas de exclusión entre los cuales deberíamos ubicar la división o la separación entre lo verdadero y lo falso.

En efecto, para Foucault, la emergencia de esta determinación discursiva que funciona en términos de separación entre lo verdadero y lo falso, constituye un acontecimiento cuya irrupción debería ser históricamente ubicada “entre Hésiodo y Platón" (FOUCAULT, 2012 [1971], p. 17) y que entre sus efectos generó ni más ni menos que la expulsión del sofista. Así, según el filósofo este advenimiento "ha sin duda dado su forma general a nuestra voluntad de saber" (FOUCAULT, 2012 [1971], p. 18).

Manteniendo esta hipótesis, durante las lecciones del 6 y del 13 de febrero de 1971 Foucault precisará entonces su postura atribuyendo a Aristóteles el golpe de gracia que marca la exclusión del sofisma del universo del discurso filosófico. Este gesto, designado como "operación apofántica", es según Foucault uno de los fenómenos que da "fundamento a la ciencia moderna y a la filosofía occidental en sus desarrollos históricos" (FOUCAULT, 2011, p. 66).

El análisis sobre las implicancias del discurso apofántico en los trabajos de Foucault y en especial de las relaciones entre éste y la conformación de la voluntad de saber excede claramente los límites de este trabajo. Sin embargo, quisiéramos observar que para Foucault, la expulsión del sofista y del sofisma del universo discursivo filosófico trae aparejada la exclusión de la materialidad del discurso.

Dado que el sofisma se sostiene a partir de la existencia del enunciado en tanto tal, su nivel operacional está limitado al simple hecho de que las palabras hayan sido efectivamente pronunciadas. Como discurso, el sofisma es un acontecimiento irrepetible que se produce una sola vez y que tiene validez porque es una simple cosa dicha. En este sentido su validez es independiente de las categorías de "verdadero" y de "falso". Puesto que el sofisma se mantiene en el nivel del simple enunciado, como una simple cosa dicha, Foucault puede aclarar que "la 
sola cosa que manipula el sofista, el sólo ser al que él se dirige, es el ser de la cosa dicha, es el ser del enunciado en su realidad material" (FOUCAULT, 2011, p. 62).

Una forma tal de discurso, que concibe las palabras, su materialidad específica, como una cosa entre otras cosas, queda entonces por fuera del discurso apofántico, que es resumido por Foucault como una operación "que establece entre el enunciado y el ser una relación a un sólo nivel (siempre ideal) de su significación" (FOUCAULT, 2011, p. 65). En otros términos, puesto que la apofántica es productora de sentido, ella debe siempre apelar a un nivel ideal que se encuentra bien lejos del enunciado pronunciado y que pone en juego una instancia metaenunciativa que en tanto tal impide el funcionamiento del enunciado como cosa.

Ahora bien, a diferencia del episodio sofístico, la exomologesis pone en juego no el nivel discursivo, sino más bien - como hemos visto - una forma de decir la verdad que implica la materialidad de los gestos, la vida misma del penitente. El análisis de la exomologesis permite así considerar una manera de decir la verdad que no sólo no es un tipo de discurso, sino una forma de expresión que carece por completo de la puesta en funcionamiento de la palabra en cuanto ella puede servir como medio de transmisión de la verdad del individuo. En este sentido, si bien el abordaje foucaultiano muestra que dentro de la exomologesis tenía lugar la articulación verbal, esta no cumplía la función de manifestar ni las faltas ni los pensamientos, sino que ocupaba un rol junto a los otros modos de expresión dentro de la economía de una manifestación de sí dramática y teatral. En la exomologesis, la verbalización no constituía una instancia en donde estaba en juego el análisis detallado de los hechos cometidos por el pecador o de su esfera interna en tanto individuo. En este sentido, y para ser más precisos, para que la palabra se constituya como el medio por el cual se describen de manera analítica las faltas del pecador, habrá que esperar no el surgimiento de la hermenéutica de sí monástica, sino el advenimiento de la penitencia tarifada a partir del siglo VII, puesto que ella pondrá en funcionamiento un mecanismo de correspondencia entre las faltas cometidas y una grilla de "satisfacciones". 
Como lo explica Foucault, el carácter verbal de la veridicción en la penitencia tarifada es necesario dado que la declaración analítica de la falta constituye un engranaje fundamental para que pueda aplicarse un código que "ha previsto tal penitencia, tal satisfacción para tal falta" y una serie de modificaciones posibles de la satisfacción "en función de una modificación de las circunstancias de la falta" (FOUCAULT, 2012b, p. 180, nota a pie de página).

En consecuencia, la reflexión que se pone en juego a partir de la exomologesis, permite observar cómo los análisis foucaultianos del cristianismo primitivo abordan un nivel de operatividad de la manifestación de la verdad - y en particular de la manifestación de la verdad de sí - que no depende del plano discursivo. Lo que muestra el análisis foucaultiano de la exomologesis, es que el revés histórico de un acontecimiento fundamental para la constitución de la voluntad de saber occidental como la hermenéutica de sí, ha sido la exclusión de una forma no verbal de decir la verdad de sí, verdad que de ahora en adelante deberá ser mediada por el discurso y la palabra.

Ciertamente, como lo señala el mismo Foucault, este tipo de manifestaciones teatrales y no verbales de sí mismo no desaparecen del universo de las prácticas cristianas. En este sentido, el filósofo cita entre otros ejemplos las penitencias públicas que seguirán siendo realizadas hasta el siglo XV inclusive (FOUCAULT, 2012b, p. 179). Asimismo, es posible admitir que dentro de la práctica de la dirección espiritual monástica, la manifestación de la obediencia del monje comprende expresiones no verbales. Sin embargo, el lugar central que ocupaba la exomologesis como forma de veridicción de sí será ocupado, a partir de la institucionalización del monacato en la Antigüedad Tardía, por la manifestación oral de la verdad del individuo. Manifestación que pondrá a su vez en juego no ya la verdad del status del individuo como pecador, sino la esfera interna de sus pensamientos. Con el monacato desaparece el rol preponderante de la materialidad de la vida y de los gestos como expresión de la verdad de sí mismo.

Ahora bien, más allá de la exclusión histórica del rol principal de una forma no verbal de decir la verdad del universo de las técnicas cristianas, el análisis de la exomologesis permite también comprender 
una nueva "problematización" en la relación que el cristiano mantiene consigo mismo. De la penitencia canónica al nacimiento del monacato, podemos individualizar una cesura relativa a la manera en que el cristiano se vincula con su propia verdad. Para el penitente que practicaba la exomologesis, la cuestión principal en los actos y en la vida que él llevaba, consistía en manifestarse, en publicarse, como pecador. La verdad absolutamente expuesta de sí mismo constituía el núcleo de esta parte central de la penitencia. A partir del monacato, la verdad de sí pasa a ser algo que se conoce y que se interpreta, dado que el cristiano, por sí mismo, no es capaz de establecer la filiación de sus propios pensamientos. Así, uno de los principales problemas que emergen es este carácter opaco, oscuro, que marca la relación del individuo consigo mismo respecto de su propia verdad. En el origen mismo de la hermenéutica de sí, es posible ver cómo la relación consigo mismo reviste un rol capital en la instauración de la enunciación de la palabra como instrumento de separación y descrifamiento de los pensamientos: la relación consigo mismo es uno de los principales aspectos que sirven para explicar la desaparición del papel central de una manifestación de sí no verbal, en la cual la verdad no necesitaba ser interpretada.

Para resumir, la exclusión de la exomologesis como práctica central de la veridicción de sí mismo parece estar entonces marcada por estos dos fenómenos: por un lado, la desaparición de la materialidad de los gestos, de los actos y de la vida misma como canales de expresión preeminentes de la verdad del sujeto; por otro, la opacidad en la relación consigo mismo respecto del establecimiento de la verdad de sí. Estos dos elementos, ligados directamente al problema de la verdad y la subjetividad, ocupan un lugar central - junto a la necesidad de la obediencia absoluta en el marco de la dirección espiritual - en el momento en que asistimos a la instauración de la confesión (aveu) en el núcleo de las prácticas monásticas.

De este modo, preguntarse por la exclusión de la exomologesis, nos permite abordar aquello que queda en los márgenes en un momento fundamental de la historia de la confesión. Así, en el marco de una empresa que se comprende - entre otros aspectos - a partir del esfuerzo y la inquietud de encontrar los medios para "pensar de otra 
manera" (FOUCAULT, 2012 [1984], p. 16), la exclusión de la exomologesis nos recuerda que con anterioridad al monacato, en Occidente otra manera de decir la verdad ocupaba un rol central y que ella implicaba una relación del sujeto consigo mismo muy diferente de aquella que marca un acontecimiento capital en la hegemonía histórica de la confesión (aveu) en el marco de las prácticas penitenciales y de la dirección espiritual.

\section{Referencias}

CHEVALLIER, P. Michel Foucault et le christianisme. Lyon: ENS Editions, 2011.

FOUCAULT, M. Du gouvernement des vivants: Cours au Collège de France 19791980. Paris: Seuil; Gallimard; EHESS, 2012a. (Hautes études).

FOUCAULT, M. Histoire de la sexualité I: La volonté de savoir. Paris: Gallimard, [1976] 2012. (Collection TEL).

FOUCAULT, M. Historia de la sexualidad I: La voluntad de saber. Buenos Aires: Siglo XXI, 2005.

FOUCAULT, M. Histoire de la sexualité II: L'usage des plaisirs. Paris: Gallimard, [1984] 2012 . (Collection TEL).

FOUCAULT, M. Historia de la sexualidad II: El uso de los placeres. Buenos Aires. Siglo XXI, 2005.

FOUCAULT, M. Leçons sur la volonté de savoir: Cours au Collège de France 1970-1971. Paris: Seuil; Gallimard, 2011. (Hautes études).

FOUCAULT, M. Lecciones sobre la voluntad de saber: Curso en el Collège de France 1970-1971. Buenos Aires. FCE, 2012.

FOUCAULT, M. Les anormaux: Cours au Collège de France 1974-1975. Paris: Seuil; Gallimard, 1999. (Hautes études).

FOUCAULT, M. Los Anormales: Curso en el Collège de France 1974-1975. Buenos Aires: FCE, 2000. 
FOUCAULT, M. L'Ordre du discours. Paris: Gallimard, [1971] 2012.

FOUCAULT, M. El orden del discurso. Barcelona. Tusquets, 1999.

FOUCAULT, M. L'origine de l'herméneutique de soi: Conférences prononcées à Dartmouth College, 1980. Paris: Vrin, 2013. (Philosophie du présent).

FOUCAULT, M. Mal faire, dire vrai: Fonctions de l'aveu en justice. Louvain: Presses Universitaires de Louvain; Chicago University Press, 2012b.

FOUCAULT, M. Obrar mal, decir la verdad: La función de la confesión en la justicia. Buenos Aires: Siglo XXI, 2014.

Recibido: $16 / 11 / 2015$

Received: 11/16/2015

Aprobado: 20/05/2015

Approved: 05/20/2015 